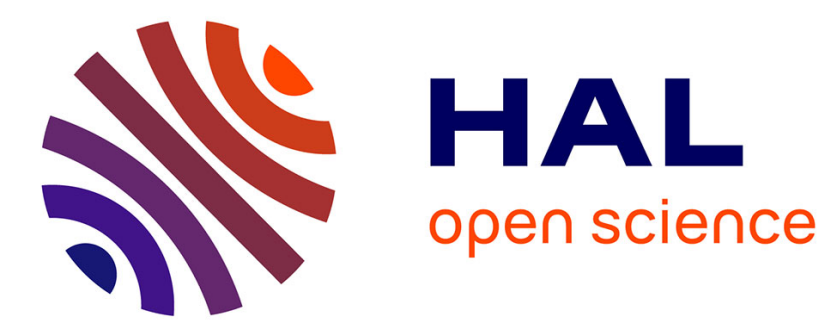

\title{
LES ENSEIGNANTS DE FRANÇAIS FACE AU CINÉMA : UN PROBLÈME DE REPRÉSENTATION?
}

Philippe Bourdier

\section{To cite this version:}

Philippe Bourdier. LES ENSEIGNANTS DE FRANÇAIS FACE AU CINÉMA: UN PROBLÈME DE REPRÉSENTATION?. Le Français Aujourd'hui, 2009. hal-03233583

\section{HAL Id: hal-03233583 \\ https://hal.science/hal-03233583}

Submitted on 25 May 2021

HAL is a multi-disciplinary open access archive for the deposit and dissemination of scientific research documents, whether they are published or not. The documents may come from teaching and research institutions in France or abroad, or from public or private research centers.
L'archive ouverte pluridisciplinaire HAL, est destinée au dépôt et à la diffusion de documents scientifiques de niveau recherche, publiés ou non, émanant des établissements d'enseignement et de recherche français ou étrangers, des laboratoires publics ou privés. 
La réflexion qui va suivre est née de plusieurs constats d'écarts parfois paradoxaux : d'une part entre les caractéristiques des pratiques effectives de l'enseignement du français dans les collèges, les lycées, et, d'autre part, ce qui est prôné ou demandé à l'égard de l'image, de l'image cinématographique et plus largement du cinéma.

Ainsi, premier constat : alors que l'image relève d'un exercice intellectuel fondamental sur la polysémie des discours à exercer en français, que cet exercice est légitimé dans les programmes depuis 1995, que des pratiques pionnières ont existé dans la marginalité des pratiques innovantes du système éducatif français depuis les années $1970^{1}$, celles-ci sont encore peu répandues dans les classes. Deuxième constat: alors que les enseignants de français intègrent généralement peu l'étude de l'image cinématographique ou des écrits de cinéma dans leurs séquences d'apprentissage, ce sont eux pourtant qui majoritairement inscrivent leurs classes aux dispositifs d'acculturation en faveur des pratiques de spectateur de cinéma, comme «Collège au cinéma », «Lycéens au cinéma ». Troisième constat : alors que l'image relève de plein droit des supports de lecture dans la didactique du français du second degré, les manuels qui s'adressent aux élèves, mais aussi à leurs enseignants, proposent le plus souvent des approches de l'image -particulièrement de l'image cinématographique- reléguées de manière significative en fin de séquence, qui peuvent apparaître aux yeux des uns et des autres comme des étapes superfétatoires du parcours d'apprentissage. Quatrième constat : alors que les enseignants de français se disent démunis scientifiquement face à l'étude des images à mener avec leurs élèves ${ }^{2}$, les cahiers de charges qui régissent la formation professionnelle des enseignants n'expriment souvent que peu ce besoin de formation, ou seulement de manière très inégale selon les académies. Enfin, dernier constat, qui porte cette fois sur les décisions institutionnelles : si l'image relève bien du domaine de connaissances à développer, en particulier dans la définition du socle commun de

\footnotetext{
1 L'on songe aux expériences de René de la Borderie de 1965 à 1976 à l'ICAV de Bordeaux, à celles de Sylvain Roumette et de Geneviève Jacquinot au collège expérimental de Marly le Roi, mais aussi, et surtout dans un article pour cette revue, au Manifeste de Charbonnières, rédigé en 1969 par les militants de 1'Association Français des Professeurs de Français. Ce manifeste exprimait notamment le souhait d'un enseignement du français qui ne se limite pas à celui de la seule littérature.

2 A $46 \%$ selon un sondage SCP communication pour le compte de l'inspection générale de Lettres en 2005. Nous signalons quelques contributions utiles à la réflexion didactique sur les rapports textes-images: l'article essentiel d'Isabelle Nières, « Des illustrations exemplaires : Max et les maximonstres », paru dans le numéro 50 en 1980 dans Le français aujourd'hui, repris dans La revue des livres pour enfants, $\mathrm{n}^{\circ}$ 232, décembre 2006, p. 129-141. Les lecteurs intéressés par ce sujet trouveront un intérêt à lire le numéro 162 , de juin 2008 de Le français aujourd'hui tout entier consacré à ce sujet, ou bien Les contes de Charles Perrault illustrés par Gustave Doré : langage verbal et image, de Pascal Caglar et Philippe Bourdier, Paris, 2006, éd. Ellipses.
} 
Philippe Bourdier

LFA n ${ }^{\circ} 165$

LES ENSEIGNANTS DE FRANÇAIS FACE AU CINÉMA : UN PROBLÈME DE REPRÉSENTATION ?

connaissances et de compétences apparu en 2006, elle reste quasiment encore absente des épreuves certificatives que peuvent connaître les professeurs de lettres.

Ces derniers écarts peuvent s'expliquer par des choix de politique éducative, il n'en est pas tout à fait de même pour les autres écarts, puisque ceux-ci relèvent de décisions individuelles plus ou moins assumées collectivement. Or, ces formes paradoxales des pratiques professionnelles peuvent correspondre au poids des représentations comme un en-deçà prégnant et structurant.

Car l'introduction de l'image dans les programmes français de l'enseignement secondaire, notamment de l'image cinématographique, nous semble, elle aussi et pour une part, tributaire des représentations que les enseignants en ont. Ainsi, quelles sont les attentes des enseignants de français à l'égard de l'appui pédagogique préconisé sur le cinéma ? Comment interprètentils la possibilité nouvelle de s'attacher à une autre forme de discours à didactiser et comment la mettent-ils en cohérence avec les missions qu'ils assignent à leur fonction ? Ces questions se posent particulièrement toutes les fois que les pratiques portent sur des objets du savoir enseigné qui sont, soit remis en question par les débats publics, soit récents dans la discipline scolaire, soit extérieurs au champ de référence de la discipline. Tel est le cas pour l'image dans la didactique du français ; tel est le sujet envisagé en ce qui concerne les enseignants de français, du seul point de vue de leurs représentations à l'égard de l'image cinématographique, plus largement du cinéma, à partir de la question centrale pour eux de la posture cognitive de l'interprétation des images.

Ainsi, à partir d'une enquête de terrain menée de 2001 à 2006, envisageons tout d'abord la signification du concept de représentation ainsi que ses résonances dans la didactique du français, pour ensuite cerner quelques caractéristiques essentielles de ces représentations qui ont trait à l'interprétation des images en cours de français.

\section{L'intérêt des représentations sociales}

Les représentations constituent un domaine d'intérêt fort marqué au sein des sciences sociales. Elles sont constituées «d'un ensemble d'informations, de croyances, d'opinions et d'attitudes à propos d'un objet donné. »(J-C. Abric, 1987 : 17). Leur étude n'a été que renforcée au fil des ans dans la mesure où celle-ci permet de comprendre ce qui relie des individus dans leurs pratiques sociales, au-delà de leurs différences apparentes et qui reposent 
Philippe Bourdier

LFA n ${ }^{\circ} 165$

LES ENSEIGNANTS DE FRANÇAIS FACE AU CINÉMA : UN PROBLÈME DE REPRÉSENTATION ?

sur des valeurs souvent fortement intégrées mais non exprimées. Toute représentation est en effet « une forme de connaissance, socialement élaborée et partagée ayant une visée pratique et concourant à la construction d'une réalité commune à un ensemble social» (D. Jodelet, $1986: 36)$.

Nombre de recherches ont porté sur des milieux socio-professionnels divers dans les années 90, mais peu ont concerné les enseignants du second degré et rares sont celles qui vont jusqu'à tirer des conclusions d'ordre didactique en examinant les choix et le traitement pédagogique des contenus enseignés qui peuvent dépendre pour une part des représentations que les enseignants ont ancrées en eux.

Quelles idées les individus portent-ils en eux de leur profession, de leurs rôles ? Quelles sont celles qu'ils partagent? Quelles sont-celles qui les différencient? Quelles sont-celles qu'ils affichent? Comment celles-ci s'articulent-elles avec les instructions officielles mais aussi avec les pratiques des collègues? Telles sont quelques questions importantes, souvent négligées dans la réflexion sur les pratiques professionnelles des enseignants, sur leur formation universitaire, sur l'analyse de leurs difficultés ou de leur épanouissement dans les classes.

En effet, il serait naïf de croire que tout enseignant n'est animé dans son exercice professionnel que par le respect scrupuleux des programmes, l'observance des règles du bon fonctionnaire ou bien par l'ambition de faire progresser ses élèves ou bien encore de reproduire, de transposer des éléments de connaissances que lui-même a pris du temps pour acquérir au cours de ses études. Il s'y mêle d'autres mobiles, tantôt liés aux circonstances dans lesquelles l'on exerce, qui relèvent de l'adaptation, tantôt liés à des conceptions de l'enseignement souvent non verbalisées en société : sa façon de concevoir l'apprentissage, son propre passé scolaire, ses propres expériences sociales, ses valeurs, sa formation, son langage et ses modes de communication, ses outils pédagogiques de prédilection et enfin ses représentations de sa discipline scolaire. L'ensemble de ces paramètres influe sur la manière d'enseigner, sur les choix des gestes professionnels, sur les attentes à l'égard des situations d'enseignement ou bien participent des «logiques d'arrière-plan » (J.C. Chabanne-D. Bucheton, 2002) qui sont à l'œuvre.

Il apparaît, de surcroît, que le poids de ces représentations est d'autant plus fort dans les choix d'enseignement que le domaine de connaissances à travailler est inédit ou nouveau pour le professeur en tant qu'objet d'analyse, mais familier en tant que champ de pratiques culturelles. Ainsi, à la différence des textes littéraires, les images cinématographiques 
semblent constituer un domaine de connaissances d'une étrange familiarité sur lequel l'enseignant doit exercer des compétences de pédagogue.

L'étude des représentations des enseignants de français à l'égard du cinéma en tant que pratique scolaire que nous avons menée repose sur une enquête de terrain qui associe une exploitation quantitative et qualitative des données. Le corpus d'étude est constitué de réponses fournies à deux questions écrites, formulées volontairement de manière directive afin d'inciter chacun à réfléchir progressivement, à la fois du côté des intérêts du cinéma en contexte scolaire, mais encore vers l'examen des obstacles à l'inscription des films dans l'enseignement du français. Ce questionnaire écrit a été complété par des entretiens semidirectifs, menés quelque temps après le dépouillement des données écrites, auprès de quarante enseignants. Ces questions écrites étaient :

- «Quels intérêts trouvez-vous à la présence de pratiques d'enseignement portant sur le cinéma et l'image cinématographique en cours de français ?

- «Selon vous, et pour vous, quelles sont les difficultés posées par les pratiques pédagogiques liées au cinéma dans vos cours?»

La présentation du questionnaire ${ }^{3}$ engageait les enseignants à répondre de manière argumentée et à produire non une réponse, mais plutôt des réponses avec des éclairages différents, ou bien des réponses en cascade avec une précision ou une complémentarité croissante. L'analyse des réponses a consisté à classer les réponses selon une typologie préétablie qui s'est affinée au fil du classement.

Les réponses apportées par écrit comme à l'oral étaient nombreuses, souvent argumentées et plus encore très diverses. Ce sont ces caractéristiques qui définissent d'abord, à nos yeux, le noyau central des représentations des enseignants de Lettres. L'on peut interpréter le mode global du traitement des questions comme le signe d'une conscience assez vive de l'intérêt de considérer la place, le rôle et les obstacles à une didactisation du cinéma dans le cadre de la classe de français. Certains (10\%) ont même souligné la rareté d'un tel domaine de

\footnotetext{
3 L'enquête de terrain a été menée auprès d'enseignants de Lettres de l'académie d'Orléans-Tours qui, pour une part, étaient volontaires et s'attendaient à réfléchir sur la didactique de l'image cinématographique (159 personnes), et pour une autre part qui n'étaient pas volontaires (79 personnes), puisqu'ils faisaient partie de ce que l'on a coutume d'appeler un «public désigné de formation » défini par les supérieurs hiérarchiques. Les questionnaires écrits furent remplis dans le cadre d'actions de formation auprès d'enseignants de français menées par l'Institut Universitaire de Formation des Maîtres du Centre Val de Loire, à la demande du rectorat. L'échantillon constitué mêlait des enseignants chevronnés (entre cinq et vingt-sept ans d'expérience, avec le plus souvent plus de cinq années dans l'un ou l'autre des cycles de l'enseignement secondaire) et des enseignants débutants, interrogés dans le cadre de leur formation initiale durant leur première année d'expérience professionnelle.
} 
Philippe Bourdier

LFA ${ }^{\circ} 165$

LES ENSEIGNANTS DE FRANÇAIS FACE AU CINÉMA : UN PROBLÈME DE REPRÉSENTATION ?

réflexion professionnelle. Sans mésestimer le poids du contexte particulier dans lequel ces interrogations furent formulées, l'ensemble des réponses témoigne d'un intérêt pour les questions de didactique de l'image, particulièrement de l'image mobile. On peut y voir aussi une ouverture intellectuelle des enseignants à ces domaines d'apprentissage inscrits dans les programmes scolaires.

Mais ouverture intellectuelle ne signifie pas nécessairement intégration aisée dans les pratiques pédagogiques. En effet, si les œuvres cinématographiques, les images cinématographiques ne sont pas perçues comme illégitimes ${ }^{4}$ dans la classe de français, suscitent même fréquemment un intérêt personnel, en revanche la définition du type d'interprétation des films, des extraits de films, des images cinématographiques à développer en classe constitue un obstacle certain, en dépit des précisions apportées par les programmes et les documents d'accompagnement.

\section{Quelles lectures des images cinématographiques?}

Les enseignants de français consultés semblent en effet entretenir une conception paradoxale de l'image cinématographique. Pour une grande part d'entre eux (74\%), cette dernière procède de choix esthétiques ; elle naît d'une composition, possède une signification qui repose sur des intentions d'expression artistique. Mais elle offre aussi un accès facile à la compréhension dans la mesure où elle appartient d'abord à la sphère de l'image, c'est-à-dire à une forme de langage où la signification serait explicite. Rares sont ceux qui procèdent à la distinction du visible et du lisible de l'image (15\%), préférant développer l'idée que le cinéma offre une vision plus immédiate que d'autres formes de symbolisme. C'est pourquoi présenter un extrait de film permet, par exemple, de fournir une illustration visuelle (15\%) d'un cadre historique, social, géographique ou psychologique d'une interprétation d'une œuvre théâtrale, voire, compense l'impossibilité d'assister à la représentation théâtrale $(10,5 \%)$. Les vertus du cinéma sont aussi liées au fait que les films donneraient à voir ce que les élèves ont habituellement à imaginer, ou permettraient de faire comprendre ce qui relève de l'ordre de la caractérisation esthétique abstraite : la préciosité, le romantisme sont ainsi souvent mentionnés.

4 Selon le sondage CSP $81 \%$ des enseignants de Français estiment plus largement que l'étude de l'image relève de l'enseignement des Lettres. 
En somme, pour beaucoup, l'image cinématographique offre des intérêts qui proviennent de l'analogie entre le texte et son image et n'est guère conçue que comme illustration de cours. Les arguments développés par Christian Metz dans les années $70^{5}$ n'ont trouvé que peu de retentissement dans le système de pensée des enseignants de français : les images, si elles contiennent de l'analogie, ne se réduisent pas à cela. Christian Metz a développé l'idée que l'image contient certes de «l'analogie» mais la concevoir ainsi serait réducteur de sa complexité. En outre l'analogie n'y sert, le plus souvent, qu'à véhiculer un message qui, lui, n'a rien d'analogique, ni même de visuel: par exemple, des visées publicitaires ou religieuses. C'est dire, donc, comme le souligne Jacques Aumont que l'analogie se trouve elle-même codée. De surcroît, l'analogie dans l'image cinématographique n'a souvent pour fonction que de permettre la communication d'un message qui, lui, n'est pas analogique et, parfois, peut ne pas être visuel : un récit par exemple. Ceux qui portent le plus solidement cette représentation strictement analogique du film partagent aussi souvent l'idée que la complexité des films peut provenir de la coexistence de plusieurs langages unifiés dans l'œuvre : «les films lient plusieurs langages dans la construction d'une émotion (musique, image) ». ${ }^{6} \mathrm{~L}$ 'articulation logique et la mise en cohérence entre le mode esthétique et le mode épistémique de l'image au sens où Jacques Aumont dans L'image, à la suite de Rudolf Arnheim, a pu le définir semble se faire dans les représentations des enseignants de français grâce au concept de «confiance abusive» que, dans tous les cas, les enseignants (42\%) avancent pour caractériser le lien qui unit élèves et images animées.

Que le film apparaisse comme une production dont la signification et les valeurs sont incomplètes sans le regard du spectateur (10\%), qu'il procède d'une composition fondée sur un parti pris d'auteur (7\%) ou bien qu'il impose une représentation du réel qui se veut fidèle à son modèle, dans tous les cas l'image cinématographique est pensée comme trompeuse (78\%). Dès lors, introduire le cinéma dans les pratiques scolaires revêt l'enjeu de briser un rapport de confiance abusive avec l'image en général. Ainsi les enseignants avancent rarement l'idée qu'il n'existe pas de regard innocent chez le spectateur (au reste, pas plus qu'il n'en existe peut-être pour le lecteur) mais particulièrement chez des élèves dont les pratiques sociales de référence sont nombreuses à l'égard de toutes les formes d'écran. Cette co-construction du sens de l'image, définie par Gombrich et les théories constructivistes dès les années 60, semble secondaire par rapport aux pouvoirs fallacieux de l'image qu'il

5 L'on songe en particulier à l'article « Au-delà de l'analogie, l'image ? » paru dans Communication n 15 , en 1970.

6 Nous adoptons la présentation entre guillemets, texte en italiques pour toutes les citations correspondant aux transcriptions des réponses des enseignants. 
conviendrait de débusquer. C'est donc moins au nom d'une considération pour le spectateur comme sujet agissant dans la réception des films, qu'en vertu d'une démystification des pouvoirs de l'image que les enseignants de français conçoivent l'intérêt de s'attacher à des films dans un cadre scolaire.

De surcroît, en pensant une didactique du film en termes d'éducation à l'image, les enseignants de français semblent peu attachés à la nécessité de faire acquérir des références culturelles qui sous-tendent néanmoins les orientations de l'enseignement secondaire (9\%). En définitive, les films et les images s'inscrivent dans un rapport postmoderne où ils sont «moins objets que sujets du regard» (L. Lavaud, 1999: 97). Cette conception peut apparaître assez contradictoire avec la perspective patrimoniale et culturelle qui prévaut dans la définition de la culture humaniste que le socle commun de connaissances et de compétences avance. En effet, la culture humaniste y est présentée comme le moyen pour les élèves d'acquérir un sentiment d'appartenance historique et culturelle grâce aux formes de continuité, de rupture, d'expression de l'identité et de l'altérité que les œuvres de l'esprit signifient. Ces dernières doivent être connues; les élèves doivent être capables de les lire mais aussi de les situer dans le temps historique pour pouvoir constituer leur propre culture, leurs opinions et pour s'ouvrir au monde.

Ces représentations, souvent peu repérables dans les réponses écrites de notre enquête, se laissent toutefois fréquemment saisir dans les entretiens, en particulier, lorsqu'il s'est agi de définir plus précisément ce que signifiait l'expression, souvent rencontrée dans les réponses au questionnaire, «le développement de l'esprit critique» à inculquer aux élèves (30\%). Celui-ci est conçu comme une première forme d'interprétation ; majoritairement comme une forme de détachement de l'impression de réalité au cinéma et d'arrachement à la participation affective favorisée par l'image filmique. Les enseignants interrogés souscrivent peu ou prou à la distinction « effet de réalité / effet de réel » que J. Pierre Oudard proposait, dès 1971, dans un article des Cahiers du cinéma ${ }^{7}$, puisqu'ils prônent un dévoilement de l'effet de réel comme démarche critique à conduire vis-à-vis des films. Il s'agit, non pas de faire croire que ce que le spectateur voit est réel et encore moins que ce qu'il voit a pu exister, mais il faut développer un esprit critique capable de concevoir que le film est un «discours constitué à des fins esthétiques », qui possède «une complexité non apparente qui procède de choix inscrits dans une logique réception-interprétation ». Cette représentation tend à confondre œuvre cinématographique et œuvre littéraire, ou plus exactement l'œuvre cinématographique

7 J. P. Oudard, Cahiers du cinéma, n²28, 1971, «L'effet de réel », p . 19-28. 
voit ses spécificités gommées sous l'emprise d'un schéma d'interprétation communément admis dans le champ des études littéraires qui définit l'œuvre en général.

Ainsi, l'impression de réalité étudiée par Henri Wallon ${ }^{8}$ ou par André Michotte ${ }^{9}$ dès la fin des années 40 dans les numéros de la Revue internationale de filmologie, examinée par Christian Metz ${ }^{10}$ dans les années 60-70, est évacuée du champ de réflexion didactique qui concerne l'image filmique. L'oubli des facteurs subjectifs et partagés qui engagent les élèves spectateurs dans un rapport d'illusion référentielle aux images cinématographiques ${ }^{11}$, la méconnaissance des indices perceptifs et psychologiques de réalité des images en mouvement, l'oubli des phénomènes de participation affective qui sont favorisés paradoxalement par l'immatérialité de l'image filmique, sont autant de raisons qui permettent de penser l'esprit critique à développer comme une forme d'affranchissement de la proximité psychique du spectateur avec le film. Ce dernier est conçu comme un corps complexe à disséquer d'emblée et non comme un corps à envisager d'abord pour être ému, pour admirer, pour s'indigner.

Les moyens de développer l'esprit critique de l'élève spectateur correspondent à l'approche de l'étude des plans (21,5\%), ces derniers représentant de toute évidence le «trait caractéristique du langage cinématographique ». La justification de l'intérêt d'une telle approche dans la construction de la culture cinématographique des élèves, comme dans celle des compétences de lecteur de film se laisse difficilement définir. Cependant, la plupart des réponses font affleurer une idée proche de ce que Jean-Marie Schaeffer appelle l' «arché » ${ }^{12}$ appliquée à la photographie : le pouvoir de conviction des images tient aussi grandement au savoir, implicite ou non, que le spectateur a sur la genèse des images. S'agissant du cinéma, si les enseignants interrogés avancent peu que l'intérêt de l'étude des plans offre la possibilité de comprendre ce que Marc Vernet appelle «l'en-deça » du plan, c'est-à-dire le hors champ, pour beaucoup en revanche, les explications soulignent l'impérieuse nécessité d'étudier au cinéma les «figures de l'absence ${ }^{13}$. Il s'agit donc de renforcer pour le jeune spectateur la conscience de «l'infranchissable écart entre la salle où il est et la scène où se

\footnotetext{
8 « L'acte perceptif et le cinéma », Revue internationale de filmologie, $\mathrm{n}^{\circ}$ 13, 1953.

9 «Le caractère de réalité des projections cinématographiques », Revue internationale de filmologie, $\mathrm{n}^{\circ}$ 3-4, 1948.

10 en particulier dans Le signifiant imaginaire, Paris, 10/18, 1977 ou dans «A propos de l'impression de réalité au cinéma », Cahiers du cinéma, $\mathrm{n}^{\circ} 166-167,1965$.

11 les conditions matérielles particulières des projections cinématographiques, selon A. Michotte et $\mathrm{H}$ Wallon.

12 J-M. Schaeffer, L'image précaire, Paris, Le Seuil, 1987.

13 M. Vernet, Figure de l'absence, Paris, Ed. de l'Etoile, 1988.
} 
déroule 1'histoire $\gg .{ }^{14}$ Une conception du film comme œuvre réclamant la coopération interprétative des spectateurs se dessine donc. Comment alors, en classe, faire s'exprimer les élèves sur leur interprétation autour d'une séquence, d'un personnage, d'un aspect particulier ou d'une signification globale de l'œuvre?

Dans les représentations des enseignants de Lettres, si conflit d'interprétation il y a, c'est entre le sens construit par l'écriture filmique et les capacités de compréhension (parfois d'attention, tout simplement : 20,5\%) de chaque jeune spectateur. Ce n'est pas en raison des différences d'interprétation faites par chacun des spectateurs (6\%). La compréhension d'un film ou son interprétation - si l'on reprend la distinction couramment établie par les psycholinguistes entre compréhension littérale et compréhension inférentielle ${ }^{15}$ - est affaire de réception personnelle, conçue de manière solitaire et silencieuse. Aussi, les enseignants de Lettres semblent-ils peu envisager spontanément des situations collectives orales où les élèves débattent de leur compréhension personnelle. En concevant la compréhension des films comme relevant exclusivement de la sphère privée, le travail oral possible avec leurs élèves est délaissé ; les professeurs interrogés vivent les échanges oraux sur les films dans la classe, sous le poids des demandes des élèves, comme des dérogations à la loi selon laquelle les impressions de spectateur relèvent seulement de l'appréciation individuelle et sont peu dignes, in fine, d'être socialisées.

Cela conduit à privilégier des formes d'exploitation pédagogique des projections cinématographiques qui confinent l'élève dans sa compréhension personnelle : questionnaire de compréhension, lecture de documents d'accompagnement des films sur la genèse des films, sur des aspects biographiques. Plus rarement, il peut s'agir d'écrits d'élaboration des films (scénario, synopsis) ou de réception, comme des critiques des films parues dans la presse qui sont le plus souvent convergentes, non contradictoires. Tels sont les documents pédagogiques et les exploitations pédagogiques mentionnés majoritairement dans notre enquête.

\footnotetext{
14 Ibid. p. 122.

15 La compréhension littérale correspond à la constitution d'un savoir objectif qui met en jeu les décodages des procédés d'écriture des textes écrits ou des films dans ce que ceux-ci peuvent avoir d'explicites ; la compréhension inférentielle fait intervenir l'histoire personnelle de chaque sujet lecteur ou spectateur dans la compréhension. Ainsi, les zones d'explicite sont inégalement et diversement éclairées en fonction de l'expérience du spectateur.
} 


\section{L'image et le cinéma, supports réconciliateurs avec d'autres apprentissages en classe de Français?}

Le cinéma semble toutefois donner du sens à l'enseignement du français dans le second degré aux yeux de ceux qui en ont la charge. L'expression de ces représentations s'effectue tantôt directement $(10,5 \%)^{16}$, mais plus souvent elle procède par justification de l'intérêt d'intégrer dans la classe des films en avançant des arguments.

Le premier d'entre eux tient à un caractère actuel du cinéma $(93,5 \%)$, considéré surtout du point de vue du medium $(47 \%)^{17}$; moins du point de vue des pratiques culturelles : le fait de considérer qu'aller au cinéma, voir des films ou des extraits de films pour mieux les comprendre, correspond à une pratique culturelle caractéristique de notre époque, que l'école doit proposer à présent, ne fait pas vraiment consensus.

Les programmes de français de collège, pour les années prochaines, demandent de recourir aux images, en particulier aux images filmiques, dans la mesure où celles-ci

« constituent pour l'enseignement en général et celui du français en particulier, une ressource précieuse à plus d'un titre : en fournissant à l'élève des représentations du monde présent et passé, elle contribue efficacement à la constitution de sa culture et de son imaginaire; elle favorise l'expression des émotions et du jugement personnel; elle peut en outre consolider l'apprentissage de méthodes d'analyse. Selon les préconisations du socle commun de connaissances et de compétences (pilier 5), « une connaissance d'œuvres cinématographiques majeures du patrimoine français, européen et mondial » est encouragée ». 18

Les programmes du lycée ${ }^{19}$, avancent l'idée d'une nécessaire

« mise en relation des textes entre eux » ainsi que celle d'une confrontation « à d'autres langages, dont le discours de l'image » que la définition des contenus d'enseignement de la terminale L rend explicite avec l'objet d'étude «langage verbal et image: littérature et langage de l'image, littérature et cinéma ».

Ces injonctions, reliées aux récentes définitions du socle commun et de l'histoire des arts, rendent complexe la problématisation de l'image en général dans la didactique du français. A

16 « les films envisagés par les élèves, lorsqu'ils sont intégrés à un programme de classe justifient l'intérêt de lire les textes », «le cinéma, s'il est présent dans la classe donne souvent du sens à l'enseignement du français, non seulement pour les élèves, mais aussi parfois pour moi ».

17 « le cinéma est un medium actuel », « cela permet de moderniser les pratiques pédagogiques », « voir des films avec des élèves permet de travailler autrement, de manière plus moderne, plus actuelle, plus en phase avec les moyens de communication qui paraissent agréables pour nos élèves ».

18 BO spécial n ${ }^{\circ} 6$ du 28/8/2008, Programmes du collège, programmes de l'enseignement du français, préambule, p. 3

19 Publiés dans le bulletin officiel $\mathrm{n}^{\circ} 41 \mathrm{du}$ 7/11/2002 : Programme de la classe de seconde générale et technologique, dans le bulletin officiel n ${ }^{\circ} 40$ du 2/11/2006 : programme d'enseignement du français en classe de première des séries générales et technologiques, dans le bulletin officiel hors série n ${ }^{\circ} 3$ du 30/8/2001 : programme de l'enseignement de littérature en classe de terminale de la série littéraire (L). 
ces redéfinitions institutionnelles, s'ajoute l'existence des opérations telles que «Collège et cinéma » et «Lycée au cinéma » (19\%) qui semble avoir construit l'idée qu'intégrer des films en classe de français correspond, peu ou prou, à une recommandation officielle. À cet égard, certains semblent louer cette demande (28\%), tandis que la majorité se montre plus circonspecte (44\%), quand elle n'est pas hostile (13\%).

Enfin, le troisième type de justification doit s'envisager dans une dialectique ancienne entre étude d'une part, qui ne se conçoit que sur le mode de l'analyse et, d'autre part, les arts du spectacle, pensés comme divertissement social partagé dans une société des loisirs. En effet, si introduire le cinéma contribue à donner du sens à l'enseignement du français, c'est en quelque sorte parce que les textes littéraires apparaîtront comme nécessairement à analyser pour être compris, tandis que les films s'accommodent d'une compréhension superficielle. Tout se passe comme si voir un film ne relevait, chez le spectateur, que de la reconnaissance, du point de vue tant émotionnel que cognitif. Le rapport à l'image, éclairé par les enseignants de français est conçu en des termes très psychologiques. La satisfaction des spectateurs provient du fait que les élèves retrouvent une expérience visuelle dans une image qui, parce qu'elle est répétitive, permet de «retrouver du connu», de «rattacher de l'inconnu à du connu » et devient dès lors maîtrisable.

Cette approche des films qui serait en quelque sorte intuitivement développée par les élèves est décrite par les enseignants interrogés au moyen d'expressions telles que : «attitudes consommatrices »; «le film pour eux n'est pas à apprécier, à comprendre ou à connaître, mais à voir, c'est-à-dire à fréquenter dans un rapport d'imposition des images aux spectateurs $\gg$. Les enseignants de français opposent une autre attitude de spectateur qui leur semble préférable de développer dans le contexte scolaire, celle d'un spectateur actif qui, sans mise en jeu des schèmes de la « remémoration $»^{20}$, mais par l'entremise de l'étude, en vient à construire une vision cohérente et sensible des films. C'est pourquoi «l'étude» apparaît diversement appréciée par les enseignants de Lettres comme modalité du rapport culturel aux œuvres cinématographiques. Tantôt elle apparaît à certains comme un nécessaire

\footnotetext{
20 La remémoration correspond chez Gombrich à la mobilisation cognitive de formes simples, les «schémas » qui servent à la mémorisation du savoir sur le réel au-delà des diverses actualisations. Ces schémas sont constitués par les attentes qui font qu'aucun regard n'est innocent. Les schémas perceptifs permettent de confronter les savoirs préalables, les capacités d'organisation de la réalité du spectateur (y compris celles qu'il a sur le cinéma) aux données iconiques précédemment engrangées à mesure que les images du film défilent.
} 
dépassement de la posture de spectateur passif (17\%), tantôt au contraire, elle semble engager les élèves dans un rapport excessivement analytique à l'œuvre $(10,5 \%)^{21}$.

Les films (visionnement d'extraits en classe, analyse d'images cinématographiques : affiches publicitaires, photogrammes sont ainsi mentionnés) apparaissent enfin comme des moyens d'engager une lecture littéraire en construisant un horizon d'attente (23\%) pour les lectures littéraires, ou bien ils constituent un moyen de prolonger (59\%) les lectures littéraires en envisageant une réappropriation du sens de l'œuvre par un moyen d'expression artistique différent, situé dans une époque généralement différente (l'adaptation cinématographique de l'œuvre littéraire). C'est-à-dire que les films semblent répondre à deux interrogations majeures que suscitent les orientations actuelles de la didactique de la lecture littéraire. Comment, au seuil de la lecture, mobiliser les attentes à l'égard du texte à envisager, mais aussi les hypothèses sur les scénarios génériques de l'œuvre, dans la construction collective des significations profondes du texte littéraire ? Comment souligner auprès des élèves qu'il existe des permanences dans l'histoire des arts (permanences de thèmes, de genres, de figures), la production littéraire n'étant qu'une des incarnations artistiques de ces permanences. Cette question ayant peu de sens pour beaucoup d'élèves, dans la mesure où la dimension historique et patrimoniale de la littérature est difficile à constituer, elle constitue une zone d'interrogation récurrente dans la réflexion des professeurs de français, particulièrement ceux de lycée. On pourra peut-être voir dans ces premières conclusions l'affleurement d'une certaine difficulté à donner un sens à l'enseignement du français dans notre société, à l'importance de l'enjeu d'une culture humaniste dans l'enseignement en général. On pourra aussi les interpréter comme révélatrices de la constitution historique d'une discipline scolaire qui doit intégrer un nouveau vecteur culturel, artistique qui représente un autre système de signes.

Cette étude, partiellement présentée dans le cadre de cet article, peut faire naître plusieurs interrogations, à la fois sur la portée des conclusions dégagées, sur les précautions nécessaires à son interprétation mais aussi sur les prolongements didactiques, pédagogiques qu'elle peut esquisser ${ }^{22}$.

\footnotetext{
21 De ce point de vue, un clivage très net existe entre les enseignants de collège qui sont peu favorables à l' «étude » et ceux qui l'envisagent plus volontiers au lycée. L'ancienneté dans l'expérience professionnelle n'apparaît pas, sur ce point précis, comme un facteur déterminant.

22 Nous renvoyons pour les prolongements didactiques et pour plus de précisions sur les questions abordées dans cet article à P. Bourdier, Un grand écran pour les Lettres : le cinéma et l'enseignement du français, Paris, L'harmattan, 2008.
} 
L'enquête de terrain n'a pas valeur de sondage représentatif, aussi faut-il se garder de toute généralisation abusive. De surcroît, deux précautions doivent être prises pour l'étude des représentations sociales : en premier lieu, celles-ci nécessitent pour être étudiées, d'être interprétées. Exprimées sous la forme de discours écrits et oraux, leur étude s'expose donc au risque souligné par Dan Sperber : « on ne décrit pas une représentation, on la paraphrase, on la traduit, on la résume, on la développe, en un mot on l'interprète. Une interprétation est une représentation d'une représentation par une autre en vertu d'une similitude de contenu » (D. Sperber, 1996 : 68). D’autre part, «si les représentations varient selon les individus, elles varient aussi chez un même individu en fonction des situations. Ce sont les éléments extérieurs qui provoquent chez un individu l'explication de ses représentations. Les représentations recueillies ne peuvent donc qu'être spécifiques à un contexte particulier» (J.F. Blin, $1997:$ 82).

En dépit de ces précautions méthodologiques, ces questions sont dignes d'être posées car elles invitent à reconsidérer l'articulation didactique entre l'étude des images et celles des textes littéraires sur le mode de l'analogie ${ }^{23}$. Elles rendent utiles les examens des choix didactiques faits dans l'offre éditoriale pour les élèves et les enseignants et nous semblent avoir le mérite de permettre une confrontation de ses propres représentations, passées souvent sous silence, à celles qui sont présentées. Enfin, elles soulignent la nécessité de former les enseignants de Lettres aux problématiques d'interprétation des images fixes mais aussi mobiles dans les cursus universitaires, bien avant qu'ils n'aient eux-mêmes à l'enseigner à leurs élèves ${ }^{24}$.

Philippe BOURDIER

Université d'Orléans

Laboratoire Ligérien de Linguistique EA-3580

IUFM Centre Val de Loire

\footnotetext{
23 De ce point de vue, on ne peut que renvoyer les lecteurs à Comprendre le cinéma et les images, sous la direction de R. Gardies, ou bien à Lire les images de cinéma de L. Jullier et M. Marie, parus l'un comme l'autre en 2007.

24 Nous renvoyons pour ces questions à L'analyse de film en question: regard, champs, lectures, (dir.) Jacqueline Nacache, éd. L'Harmattan, col. Champs visuels, Paris, 2006, ainsi qu'à Un grand écran pour les Lettres : le cinéma et l'enseignement du Français, éd. L'Harmattan, col. Nouvelles Pédagogies, Paris, 2008.
} 


\section{Bibliographie}

ABRIC, J.C. (1987), Coopération, compétition et représentations sociales, Cousset, Del Val. AUMONT, J. (2000), L'image, Paris, Nathan.

BLIN, J-F. (1997), Représentations, pratiques et identités professionnelles, Paris, L'Harmattan.

BOURDIER, Ph. (2008), Un grand écran pour les Lettres : le cinéma et l'enseignement du Français, Paris, L'Harmattan, col. Nouvelles Pédagogies.

BOURDIER, Ph., CAGLAR, P. (2006), Les contes de C. Perrault illustrés par G. Doré : langage verbal et images, Paris, éd. Ellipses.

CHABANNE, J-C., BUCHETON, D. (2002), Parler et écrire pour penser, apprendre et se construire, Paris, Presses Universitaires de France.

GARDIES, R., (dir.) (2007), Comprendre le cinéma et les images, Paris, Armand Colin.

JODELET, D. (1989), Les représentations sociales, Paris, Presses Universitaires de France.

JULLIER, L., MARIE, M. (2007), Lire les images de cinéma, Paris, Larousse.

LAVAUD, L. (1999), L’image, Paris, GF Flammarion.

NACACHE, J., (dir.) (2006), L'analyse de film en question : regard, champs, lectures, Paris, 2006, éd. L'Harmattan.

NIERES, I., « Des illustrations exemplaires : Max et les maximonstres », in Le français aujourd'hui, $1980, \mathrm{n}^{\circ} 50$, repris dans La revue des livres pour enfants, $\mathrm{n}^{\circ} 232$, décembre 2006, p. 129-141.

OUDARD, J-P. (1971), « L'effet de réel », Les cahiers du cinéma, n 228, pp.19-28.

SCHAEFER, J-M. (1987), L'image précaire, Paris, Le Seuil.

SPERBER, D. (1996), La contagion des idées, Paris, Odile Jacob.

VERNET, M. (1988), Figure de l'absence, Paris, Editions de l'Etoile.

\section{Résumé :}

L'article définit les représentations que les professeurs de français ont actuellement à l'égard du cinéma en contexte scolaire à partir d'un travail de recherche sociologique, mené auprès de vastes échantillons d'enseignants de français entre 2002 et 2006. Il s'agit moins de cerner ce qui doit être fait dans les classes, que de mettre à jour ce qui est prégnant dans les esprits des enseignants de français et qui gouverne souvent subrepticement les choix professionnels.

Les conclusions de cette recherche sont mises en correspondance avec les attentes institutionnelles à l'égard du travail sur l'image, lui-même inscrit plus largement dans le socle commun des connaissances et des compétences.

\section{Mots clefs :}


Philippe Bourdier

LFA n ${ }^{\circ} 165$

LES ENSEIGNANTS DE FRANÇAIS FACE AU CINÉMA : UN PROBLÈME DE REPRÉSENTATION ?

Représentations professionnelles

Image

Cinéma

Didactique du français 